Article

\title{
Simultaneous Characterization and Quantification of Varied Ingredients from Sojae semen praeparatum in Fermentation Using UFLC-TripleTOF MS
}

\author{
Chuan Chai ${ }^{\circledR}$, Xiaobing Cui, Chenxiao Shan, Sheng Yu, Xinzhi Wang and Hongmei Wen * \\ School of Pharmacy, Nanjing University of Chinese Medicine, Nanjing 210029, Jiangsu, China; \\ echo_0523@hotmail.com (C.C.); xiaobingcui@163.com (X.C.); thomastiger@163.com (C.S.); \\ yusheng1219@163.com (S.Y.); wxzatnj@sina.com (X.W.) \\ * Correspondence: njwenhm@126.com; Tel./Fax: +86-25-8581-1839
}

Academic Editors: Alessandra Gentili and Chiara Fanali

Received: 23 April 2019; Accepted: 14 May 2019; Published: 15 May 2019

\begin{abstract}
Systematic comparison of active ingredients in Sojae semen praeparatum (SSP) during fermentation was performed using ultra-fast liquid chromatography (UFLC)-TripleTOF MS and principal component analysis (PCA). By using this strategy, a total of 25 varied compounds from various biosynthetic groups were assigned and relatively quantified in the positive or negative ion mode, including two oligosaccharides, twelve isoflavones, eight fatty acids, $\mathrm{N}$-(3-Indolylacetyl)-DL-aspartic acid, methylarginine, and sorbitol. Additionally, as the representative constituents, six targeted isoflavones were sought in a targeted manner and accurately quantified using extracted ion chromatograms (XIC) manager (AB SCIEX, Los Angeles, CA, USA) combined with MultiQuant software (AB SCIEX, Los Angeles, CA, USA). During the fermentation process, the relative contents of oligoses decreased gradually, while the fatty acids increased. Furthermore, the accurate contents of isoflavone glycosides decreased, while aglycones increased and reached a maximum in eight days, which indicated that the ingredients converted obviously and regularly throughout the SSP fermentation. In combination with the morphological changes, which meet the requirements of China Pharmacopoeia, this work suggested that eight days is the optimal time for fermentation of SSP from the aspects of morphology and content.
\end{abstract}

Keywords: Sojae semen praeparatum (SSP); fermentation; conversion; ultra-fast liquid chromatography (UFLC)-TripleTOF MS; principal component analysis (PCA)

\section{Introduction}

Fermentation is one of the major processes used in the production of food from soybeans and has played an important role in human life for centuries [1,2]. Many studies have reported the components that are converted and how bioactivities increased in soybean products during the fermentation process [3-7].

Sojae semen praeparatum (SSP), whose Chinese herbal name is dandouchi, which is a product of Chinese fermented preparation obtained from the ripe seed of soybean (Glycine max (L.) Merr.), has been used as an important component in traditional diets and as an effective traditional Chinese medicine (TCM) among the Chinese community worldwide. More people are expected to consume SSP if the fermentation process includes quality assessment and quality control. Other studies have focused on the active ingredients in SSP, such as isoflavone [8-11], peptides [12], biogenic amines [13,14], and volatile components [15], the physiological properties of SSP such as anti-oxidative activity [16], anti-proliferative activity [17], anti- $\alpha$-glucosidase activity [18] and anti-hypertensive effects [19], and species and quantities of fermenting bacteria in SSP spontaneous fermentation, such 
as bacterial fermentation and fungus fermentation $[20,21]$. However, no systematic comparison has been conducted of the active ingredients among the raw materials and the SSP products collected at different fermentation stages.

Isoflavones were reported to be representative constituents affecting soybean due to their significant estrogen-like bioactivity [22,23], which increased after fermentation [24,25]. The main isoflavones found in soybean are daidzein, genistein and glycitein, which are present either in glycosidic or aglycone form, mainly with $\beta$-glycosides and some 6"-O-malonyl or 6"-O-acetylglucose [26,27]. Aglyca were reported to have more bioactivity compared to the corresponding glycosides [28]. Some studies suggested that bacterial or fungal $\beta$-glycosidases are attractive candidates for use in converting $\beta$-glycosides isoflavone to their aglycones, thus enhancing the nutritional value of soy products $[29,30]$. However, the composition and contents change trends of isoflavones contained in SSP during fermentation have not yet been reported.

Therefore, we aimed to characterize the conversion of ingredients associated with the SSP fermentation process using ultra-fast liquid chromatography-triple time of flight mass spectrometry (UFLC-TripleTOF MS) [31], and accurately quantify the major components that vary using extracted ion chromatograms (XIC) manager (AB SCIEX, Los Angeles, CA, USA) with standard injections, thereby providing some technological supports for the optimization and quality control of SSP fermentation.

\section{Results and Discussion}

\subsection{Morphologic Changes}

Morphological changes in soybean and SSP products during fermentation were shown in Figure 1. With the increase in fermentation time, black soybean was overgrown with white hyphae, which then changed to yellow in SSP fermented for six days (S6), turned yellow completely in SSP fermented for eight days (S8), and finally hardened. In accordance with the 2015 edition of China Pharmacopoeia [32], moisturized soybeans should be fermented with boiled Artemisiae annuae herba and Mori folium until "yellow cladding". The morphological changes in S8 were consistent with these requirements, so we speculated that eight days is the optimal time for the fermentation of SSP.

\subsection{Qualitative Analysis and Principle Component Analysis (PCA)}

Using UFLC-TripleTOF MS analysis, information on intact precursors and fragment ions were obtained from a single injection.

The base peak chromatograms (BPCs) of soybean and S8 using both positive and negative ion modes are shown in Figure 2. The BPCs of S8 were significantly different from those of soybeans. Compared to the BPC of soybean in positive ion mode (Figure 2A), the BPC of S8 showed much higher peak intensities at $t_{R}=10-20 \mathrm{~min}$ (Figure 2C). Much higher peak intensities occurred at $t_{R}=0-10 \mathrm{~min}$ of the BPC for S8 (Figure 2D) in negative ion mode compared to soybean (Figure 2B), which shows that the ingredients converted during the SSP fermentation.

Figure 3A shows that the score plot of soybean and SSP products in positive ion mode is separated into three significant clusters $(P<0.05)$ for the first and the second principal components (PCs). Here, the green cluster (S2 (SSP fermented for two days), S4 (SSP fermented for four days) and S6) and the red cluster (S8, S10 (SSP fermented for ten days) and S15 (SSP fermented for fifteen days)) are separated by the first PC, whereas the blue cluster (soybean and S0) and the green cluster (S2, S4 and S6) are separated by the second PC. The first and second PCs' values are both $14.5 \%$.

Similarly, Figure 3C illustrates the score plot of soybean and SSP products in negative ion mode, which is separated into three significant clusters for the first and the second PCs. The blue cluster (soybean) and the red cluster (S6, S8, S10 and S15) are separated by the first PC, whereas the blue cluster (soybean) and the green cluster (S0, S2 and S4) are separated by the second PC. The first and second PCs' values are both $14.5 \%$. 
A

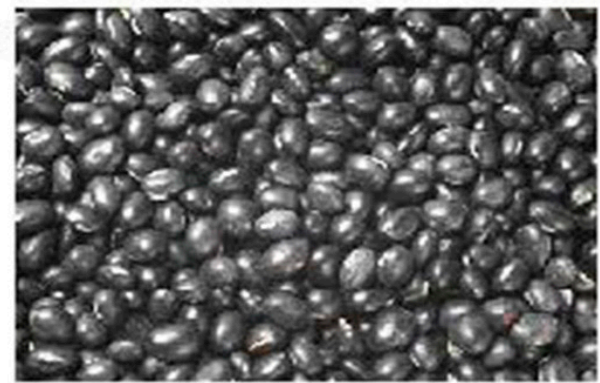

C

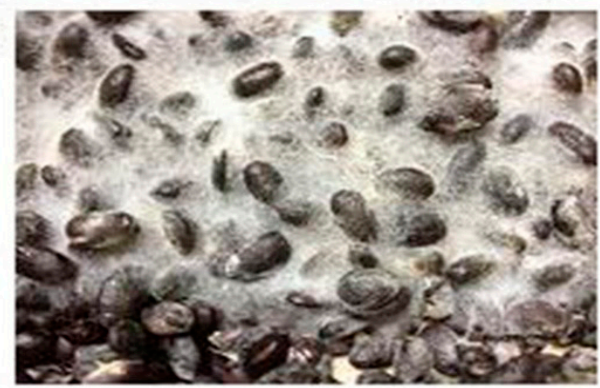

$\mathrm{E}$

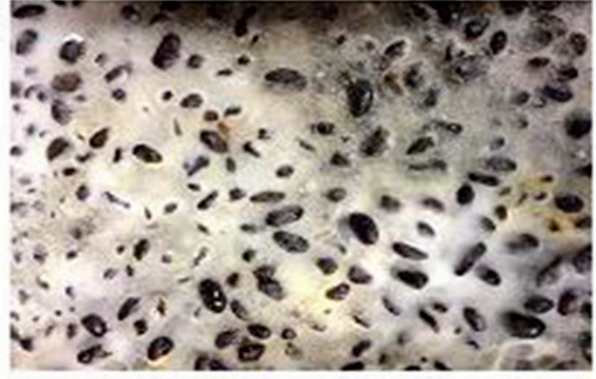

G

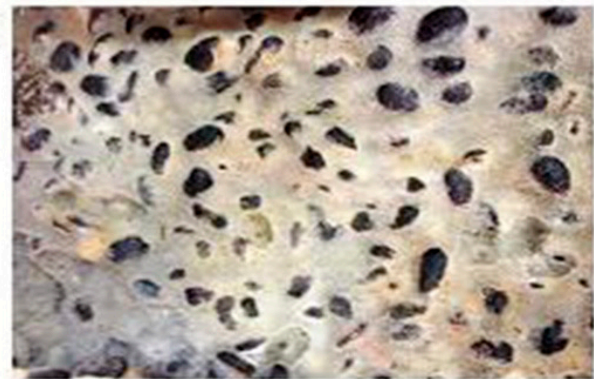

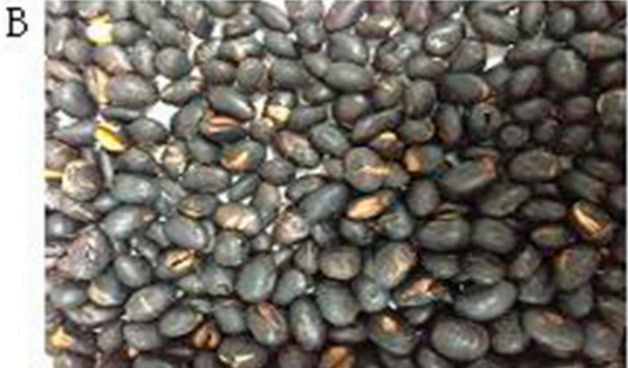

D

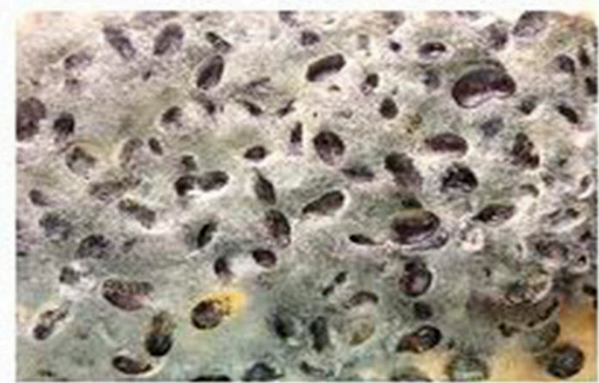

$\mathrm{F}$

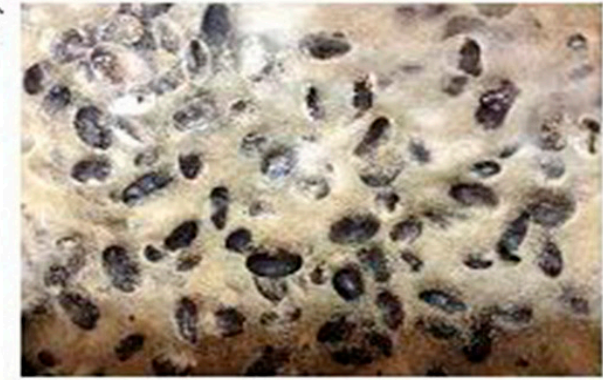

$\mathrm{H}$

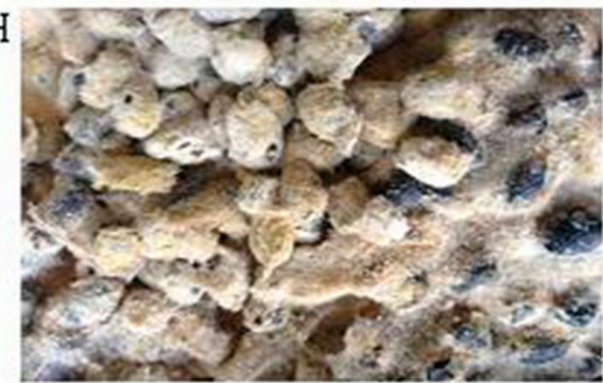

Figure 1. The morphology of soybean (A), S0 (B), S2 (C), S4 (D), S6 (E), S8 (F), S10 (G), S15 (H) days.

From the corresponding loadings plots (Figure 3B,D), a significant number of variables are located around the observations of the samples, indicating that SSP converted significantly throughout the fermentation process.

The ion species, retention times, molecular formulas, mean measured mass, mass accuracies and assigned identities of the significantly variables are shown in Table $1 .[\mathrm{M}+\mathrm{H}]^{+},[\mathrm{M}+\mathrm{Na}]^{+},[\mathrm{M}+\mathrm{K}]^{+}$, $\left[\mathrm{M}+\mathrm{NH}_{4}\right]^{+},\left[\mathrm{M}+\mathrm{H}+\mathrm{CH}_{3} \mathrm{OH}\right]^{+}$, and $\left[\mathrm{M}+\mathrm{H}-\mathrm{H}_{2} \mathrm{O}\right]^{+}$ion species were found in positive ion mode and $[\mathrm{M}-\mathrm{H}]^{-},\left[\mathrm{M}-\mathrm{H}-\mathrm{H}_{2} \mathrm{O}\right]^{-},\left[\mathrm{M}+\mathrm{CH}_{3} \mathrm{COO}\right]^{-}$, and $\left[\mathrm{M}+\mathrm{HCOO}^{-}\right.$were found in negative ion mode. 

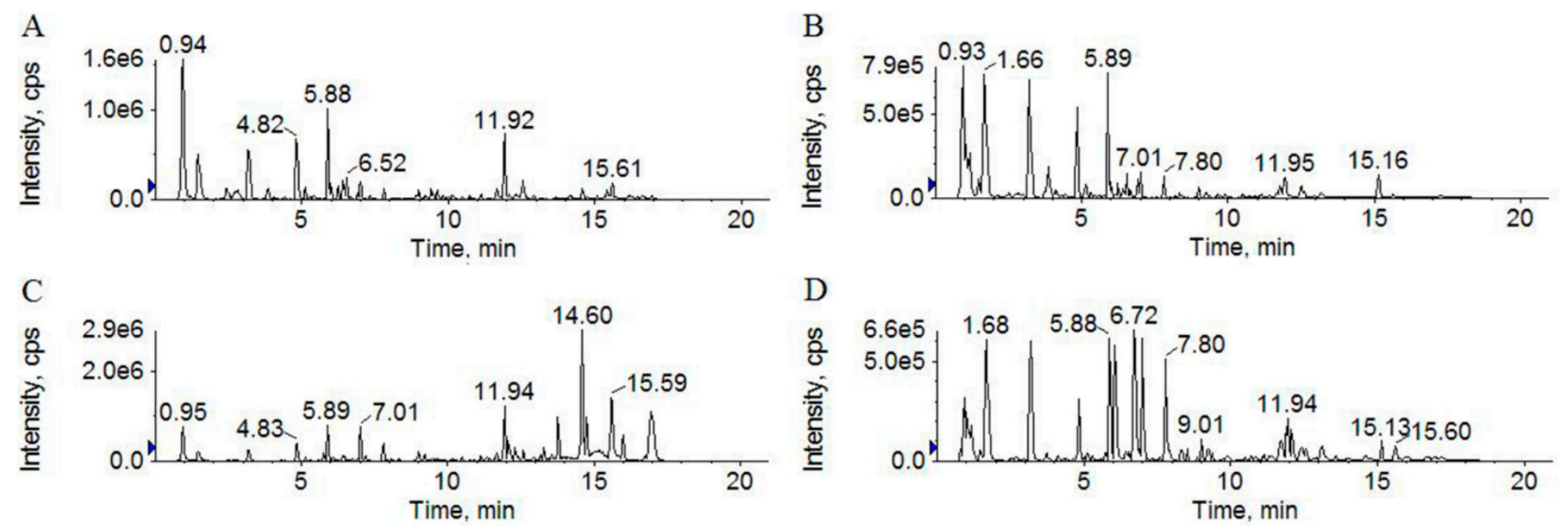

Figure 2. UFLC-TripleTOF MS base peak chromatograms (BPCs) of soybean in positive (A) and negative (B) ion modes and S8 in positive (C) and negative (D) ion modes. 
A Scores for $\mathrm{D} 1(14.5 \%)$ versus D2 (14.5 $\$$ ), Pareto (DA
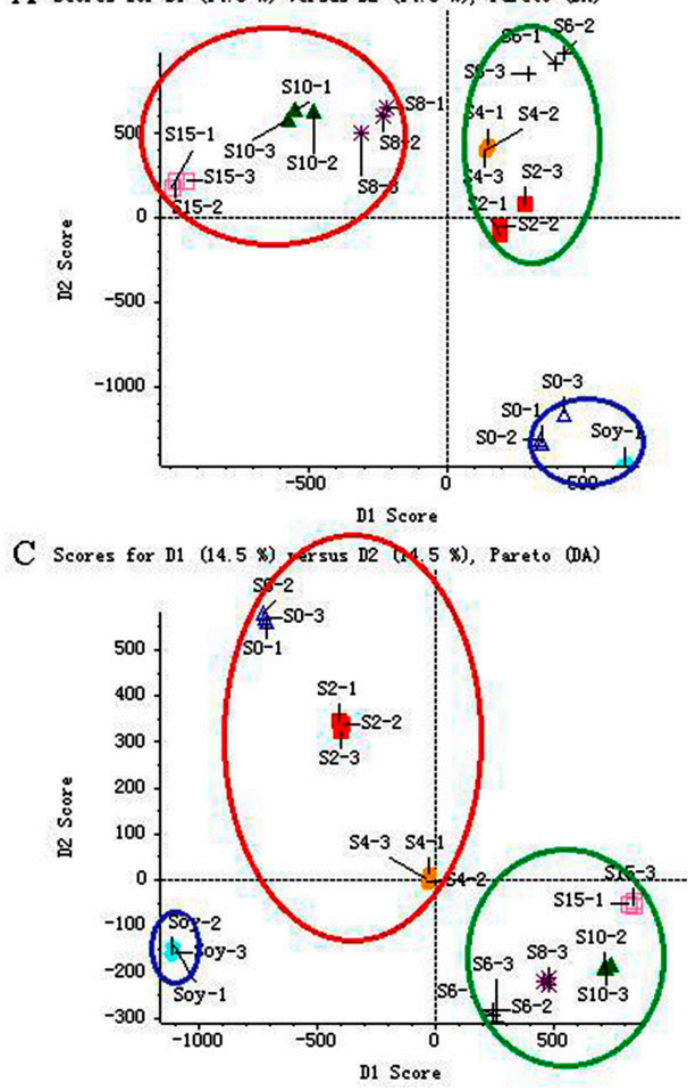

B Loadings for D1 (14.5 \$) versus D2 (14.5 \$), Pareto (DA)

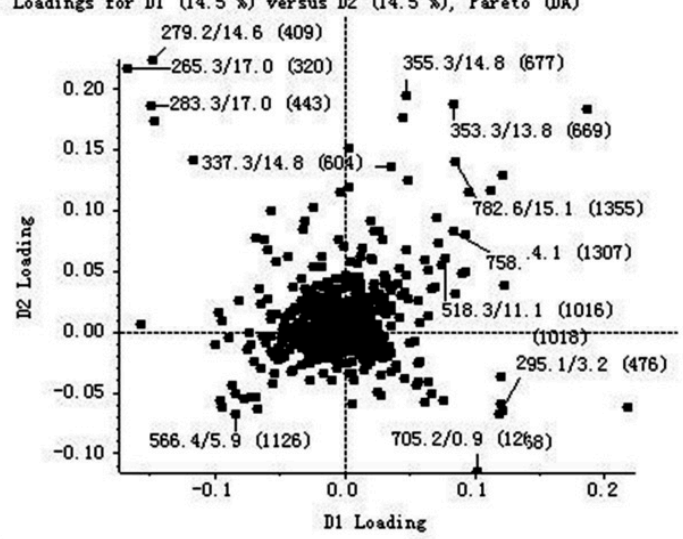

D Loadings for D1 (14.5 x) versus D2 (14.5 $x$ ), Pareto (DA) 377. $1 / 1$ : 0 (498)

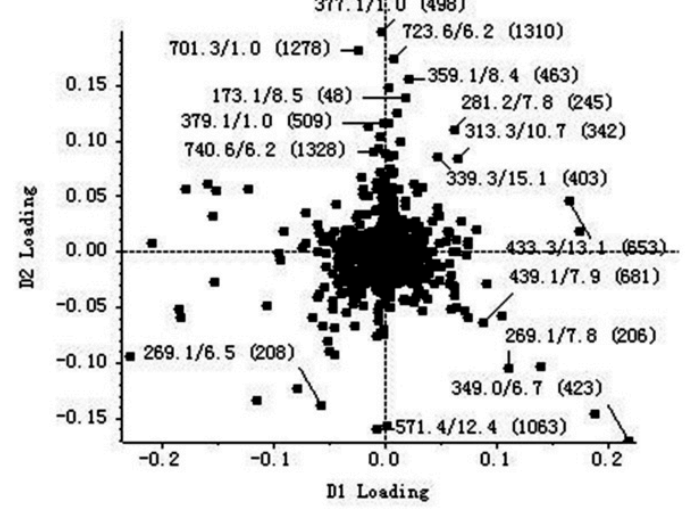

Figure 3. Score plots $(\mathbf{A}, \mathbf{C})$ and loading plots (B,D) of metabolites determined in soybean (Soy) and Sojae semen praeparatum products (S0, S2, S4, S6, S8, S10 and S15) by UFLC-TripleTOF MS in both positive ( $\mathrm{A}$ and $\mathrm{B}$ ) and negative ( $\mathrm{C}$ and $\mathrm{D}$ ) ion modes. The three clusters (green, blue and red) were used for the color coding of different groups separated by the first and second principal components.

We found 29 components of varied classes in positive ion mode, and 22 of them were inferred to be raffinose (2), stachyose (3), $N$-(3-Indolylacetyl)-DL-aspartic acid (5), daidzin (6), glycitin (7), genistin (8), 6"-O-malonyldaidzin (9), 6"-O-malonylglycitin (10), 6"-O-acetyldaidzin (11), 6"-O-acetylglycitin (12), 6"-O-malonylgenistin (13), daidzein (14), 6"-O-acetylgenistin (15), glycitein (16), methylarginine (17), genistein (18), dimorphecolic acid (20), $\alpha$-linolenic acid (22), linoleic acid (24), oleic acid (25), palmitic acid (27) and stearic acid (28) with the help of Peakview ${ }^{\circledR}$ software (AB SCIEX, Los Angeles, CA, USA). We identified 23 of varied classes in negative ion mode, of which 10 were putatively identified as stachyose (3), sorbitol (32), N-(3-Indolylacetyl)-DL-aspartic acid (5), daidzin (6), genistin (8), 6"-O-acetyldaidzin (11), 6"-O-acetylgenistin (15), glycitein (16), gheddic acid (42) and nonadecanoic acid (43) by linking the masses of ions to structures. We found 7 assigned and 1 unassigned variable in both positive and negative ion modes.

All chemical structures, selected ion intensity trend plots, mass spectra, and mass spectral interpretation of putatively assigned identities were listed in Figure 4, Figures S1 and S2, and Table 2. 
Table 1. Varied components putatively identified from soybean and Sojae semen praeparatum products in both positive and negative ion modes.

\begin{tabular}{|c|c|c|c|c|c|c|c|c|c|}
\hline $\begin{array}{l}\text { Ionization } \\
\text { Mode }\end{array}$ & $\begin{array}{l}\text { Compound } \\
\text { No. }\end{array}$ & $t_{R}{ }^{a}(\min )$ & $\begin{array}{l}\text { Molecular } \\
\text { Formula }\end{array}$ & Mass (Da) & Ion Species & $\begin{array}{l}\text { Mean Measured } \\
\text { Mass (Da) }\end{array}$ & $\begin{array}{l}\text { Mass Accuracy } \\
(\mathrm{ppm})\end{array}$ & Assigned Identity & References \\
\hline \multirow{28}{*}{ Positive } & 1 & 0.93 & $\mathrm{C}_{12} \mathrm{H}_{18} \mathrm{~N}_{6} \mathrm{O}_{6}$ & 342.1288 & {$[\mathrm{M}+\mathrm{K}]^{+}$} & 381.0907 & -0.4 & $/ \mathrm{b}$ & $-{ }^{c}$ \\
\hline & 2 & 0.93 & $\mathrm{C}_{18} \mathrm{H}_{32} \mathrm{O}_{16}$ & 504.1690 & {$[\mathrm{M}+\mathrm{K}]^{+}$} & 543.1474 & -1.9 & Raffinose & [33] \\
\hline & 3 & 0.95 & $\mathrm{C}_{24} \mathrm{H}_{42} \mathrm{O}_{21}$ & 666.2213 & {$[\mathrm{M}+\mathrm{K}]^{+}$} & 705.2045 & -1.2 & Stachyose & [33] \\
\hline & 4 & 1.44 & $\mathrm{C}_{13} \mathrm{H}_{15} \mathrm{~N}_{3} \mathrm{O}_{5}$ & 293.1012 & {$\left[\mathrm{M}+\mathrm{NH}_{4}\right]^{+}$} & 311.1328 & -2.2 & $/^{b}$ & $-c$ \\
\hline & 5 & 3.86 & $\mathrm{C}_{14} \mathrm{H}_{14} \mathrm{~N}_{2} \mathrm{O}_{5}$ & 290.0903 & {$[\mathrm{M}+\mathrm{H}]^{+}$} & 291.0955 & -4.6 & $\begin{array}{l}N \text {-(3-Indolylacetyl)- } \\
\text { DL-aspartic acid }\end{array}$ & $-{ }^{c}$ \\
\hline & 6 & 4.81 & $\mathrm{C}_{21} \mathrm{H}_{20} \mathrm{O}_{9}$ & 416.1107 & {$[\mathrm{M}+\mathrm{H}]^{+}$} & 417.1307 & 0.8 & Daidzin & [37] \\
\hline & 7 & 5.14 & $\mathrm{C}_{22} \mathrm{H}_{22} \mathrm{O}_{10}$ & 446.1213 & {$[\mathrm{M}+\mathrm{H}]^{+}$} & 447.1429 & -0.3 & Glycitin & [37] \\
\hline & 8 & 5.89 & $\mathrm{C}_{21} \mathrm{H}_{20} \mathrm{O}_{10}$ & 432.1057 & {$[\mathrm{M}+\mathrm{H}]^{+}$} & 433.1266 & 0.9 & Genistin & [37] \\
\hline & 9 & 6.03 & $\mathrm{C}_{24} \mathrm{H}_{22} \mathrm{O}_{12}$ & 502.1111 & {$[\mathrm{M}+\mathrm{H}]^{+}$} & 503.1335 & -0.6 & $6^{\prime \prime}-O-$ malonyldaidzin & [37] \\
\hline & 10 & 6.04 & $\mathrm{C}_{25} \mathrm{H}_{24} \mathrm{O}_{13}$ & 532.1217 & {$[\mathrm{M}+\mathrm{H}]^{+}$} & 533.1451 & -1.3 & $6^{\prime \prime}-O-$ malonylglycitin & [37] \\
\hline & 11 & 6.44 & $\mathrm{C}_{23} \mathrm{H}_{22} \mathrm{O}_{10}$ & 458.1213 & {$[\mathrm{M}+\mathrm{H}]^{+}$} & 459.1435 & -0.2 & 6"-O-acetyldaidzin & [37] \\
\hline & 12 & 6.51 & $\mathrm{C}_{24} \mathrm{H}_{24} \mathrm{O}_{11}$ & 488.1319 & {$[\mathrm{M}+\mathrm{H}]^{+}$} & 489.1535 & -1.0 & 6"-O-acetylglycitin & [37] \\
\hline & 13 & 6.57 & $\mathrm{C}_{24} \mathrm{H}_{22} \mathrm{O}_{13}$ & 518.1060 & {$[\mathrm{M}+\mathrm{H}]^{+}$} & 519.1285 & -0.8 & $6^{\prime \prime}-O-$ malonylgenistin & [37] \\
\hline & 14 & 7.00 & $\mathrm{C}_{15} \mathrm{H}_{10} \mathrm{O}_{4}$ & 254.0579 & {$[\mathrm{M}+\mathrm{H}]^{+}$} & 255.0726 & 0.9 & Daidzein & [37] \\
\hline & 15 & 7.05 & $\mathrm{C}_{23} \mathrm{H}_{22} \mathrm{O}_{11}$ & 474.1162 & {$[\mathrm{M}+\mathrm{H}]^{+}$} & 475.1379 & -1.0 & $6^{\prime \prime}-O-$ acetylgenistin & [37] \\
\hline & 16 & 7.17 & $\mathrm{C}_{16} \mathrm{H}_{12} \mathrm{O}_{5}$ & 284.0685 & {$[\mathrm{M}+\mathrm{H}]^{+}$} & 285.0840 & 0.3 & Glycitein & [37] \\
\hline & 17 & 7.69 & $\mathrm{C}_{7} \mathrm{H}_{16} \mathrm{~N}_{4} \mathrm{O}_{2}$ & 188.1268 & {$\left[\mathrm{M}+\mathrm{H}+\mathrm{H}_{2} \mathrm{O}\right]^{+}$} & 207.1452 & -3.9 & Methylarginine & $-{ }^{c}$ \\
\hline & 18 & 7.80 & $\mathrm{C}_{15} \mathrm{H}_{10} \mathrm{O}_{5}$ & 270.0528 & {$[\mathrm{M}+\mathrm{H}]^{+}$} & 271.0680 & 1.0 & Genistein & [37] \\
\hline & 19 & 11.94 & $\mathrm{C}_{21} \mathrm{H}_{45} \mathrm{~N}_{9} \mathrm{O}_{6}$ & 519.3493 & {$[\mathrm{M}+\mathrm{H}]^{+}$} & 520.3534 & 1.0 & $/ \mathrm{b}$ & $-c$ \\
\hline & 20 & 13.29 & $\mathrm{C}_{18} \mathrm{H}_{32} \mathrm{O}_{3}$ & 296.2710 & {$[\mathrm{M}+\mathrm{H}]^{+}$} & 297.2517 & -3.9 & Dimorphecolic acid & $-{ }^{c}$ \\
\hline & 21 & 13.77 & $\mathrm{C}_{23} \mathrm{H}_{44} \mathrm{O}_{2}$ & 352.3336 & {$[\mathrm{M}+\mathrm{H}]^{+}$} & 353.2797 & 0.1 & $/ \mathrm{b}$ & $-{ }^{c}$ \\
\hline & 22 & 14.61 & $\mathrm{C}_{18} \mathrm{H}_{30} \mathrm{O}_{2}$ & 278.2246 & {$[\mathrm{M}+\mathrm{H}]^{+}$} & 279.2408 & -0.4 & $\alpha$-Linolenic acid & [34] \\
\hline & 23 & 14.76 & $\mathrm{C}_{23} \mathrm{H}_{46} \mathrm{O}_{2}$ & 354.3492 & {$[\mathrm{M}+\mathrm{H}]^{+}$} & 355.2950 & 0.8 & $/^{\mathrm{b}}$ & $-c$ \\
\hline & 24 & 15.62 & $\mathrm{C}_{18} \mathrm{H}_{32} \mathrm{O}_{2}$ & 280.2402 & {$[\mathrm{M}+\mathrm{H}]^{+}$} & 281.2567 & 0.7 & Linoleic acid & [34] \\
\hline & 25 & 15.70 & $\mathrm{C}_{18} \mathrm{H}_{34} \mathrm{O}_{2}$ & 282.2559 & {$\left[\mathrm{M}+\mathrm{H}+\mathrm{CH}_{3} \mathrm{OH}\right]^{+}$} & 315.2915 & 0.6 & Oleic acid & [34] \\
\hline & 26 & 16.01 & $\mathrm{C}_{21} \mathrm{H}_{40} \mathrm{O}_{2}$ & 324.3023 & {$\left[\mathrm{M}+\mathrm{H}+\mathrm{CH}_{3} \mathrm{OH}\right]^{+}$} & 357.3101 & 2.9 & $/^{b}$ & $-c$ \\
\hline & 27 & 16.67 & $\mathrm{C}_{16} \mathrm{H}_{32} \mathrm{O}_{2}$ & 256.2402 & {$[\mathrm{M}+\mathrm{H}]^{+}$} & 257.2565 & -0.3 & Palmitic acid & [34] \\
\hline & 28 & 17.04 & $\mathrm{C}_{18} \mathrm{H}_{36} \mathrm{O}_{2}$ & 284.2715 & {$[\mathrm{M}+\mathrm{H}]^{+}$} & 285.3050 & -0.8 & Stearic acid & [34] \\
\hline \multirow{6}{*}{ Negative } & 29 & 0.92 & $\mathrm{C}_{12} \mathrm{H}_{18} \mathrm{~N}_{6} \mathrm{O}_{6}$ & 342.1288 & {$\left[\mathrm{M}+\mathrm{HCOO}^{-}\right.$} & 387.1623 & 1.7 & $/^{b}$ & $-{ }^{c}$ \\
\hline & 3 & 0.95 & $\mathrm{C}_{24} \mathrm{H}_{42} \mathrm{O}_{21}$ & 666.2213 & {$\left[\mathrm{M}+\mathrm{HCOO}^{-}\right.$} & 711.3077 & 1.3 & Stachyose & [33] \\
\hline & 30 & 1.05 & $\mathrm{C}_{30} \mathrm{H}_{32} \mathrm{~N}_{6} \mathrm{O}_{9}$ & 620.2220 & {$\left[\mathrm{M}-\mathrm{H}-\mathrm{H}_{2} \mathrm{O}\right]^{-}$} & 601.2133 & -1.1 & $/^{b}$ & $-c$ \\
\hline & 31 & 3.12 & $\mathrm{C}_{16} \mathrm{H}_{18} \mathrm{~N}_{6}$ & 294.1582 & {$[\mathrm{M}-\mathrm{H}]^{-}$} & 293.1552 & -1.4 & $/^{b}$ & $-{ }^{c}$ \\
\hline & 32 & 3.21 & $\mathrm{C}_{6} \mathrm{H}_{14} \mathrm{O}_{6}$ & 182.0790 & {$\left[\mathrm{M}+\mathrm{CH}_{3} \mathrm{COO}\right]^{-}$} & 241.0915 & 0.8 & Sorbitol & [35] \\
\hline & 5 & 3.85 & $\mathrm{C}_{14} \mathrm{H}_{14} \mathrm{~N}_{2} \mathrm{O}_{5}$ & 290.0903 & {$[\mathrm{M}-\mathrm{H}]^{-}$} & 289.1078 & -1.3 & $\begin{array}{l}N \text {-(3-Indolylacetyl)- } \\
\text { DL-aspartic acid }\end{array}$ & $-{ }^{c}$ \\
\hline
\end{tabular}


Table 1. Cont.

\begin{tabular}{|c|c|c|c|c|c|c|c|c|c|}
\hline $\begin{array}{l}\text { Ionization } \\
\text { Mode }\end{array}$ & $\begin{array}{c}\text { Compound } \\
\text { No. }\end{array}$ & $t_{R}{ }^{a}(\min )$ & $\begin{array}{l}\text { Molecular } \\
\text { Formula }\end{array}$ & Mass (Da) & Ion Species & $\begin{array}{c}\text { Mean Measured } \\
\text { Mass (Da) }\end{array}$ & $\begin{array}{l}\text { Mass Accuracy } \\
\text { (ppm) }\end{array}$ & Assigned Identity & References \\
\hline \multirow{18}{*}{ Negative } & 6 & 4.81 & $\mathrm{C}_{21} \mathrm{H}_{20} \mathrm{O}_{9}$ & 416.1107 & {$\left[\mathrm{M}+\mathrm{HCOO}^{-}\right.$} & 461.1650 & 0.2 & Daidzin & [37] \\
\hline & 33 & 5.64 & $\mathrm{C}_{18} \mathrm{H}_{10} \mathrm{~N}_{2} \mathrm{O}_{6}$ & 350.0528 & {$[\mathrm{M}-\mathrm{H}]^{-}$} & 349.0451 & -2 & $/ \mathrm{b}$ & $-c$ \\
\hline & 8 & 5.89 & $\mathrm{C}_{21} \mathrm{H}_{20} \mathrm{O}_{10}$ & 432.1057 & {$\left[\mathrm{M}+\mathrm{HCOO}^{-}\right.$} & 477.1629 & -2.5 & Genistin & {$[37]$} \\
\hline & 34 & 6.15 & $\mathrm{C}_{18} \mathrm{H}_{4} \mathrm{~N}_{6}$ & 304.0497 & {$\left[\mathrm{M}+\mathrm{CH}_{3} \mathrm{COO}\right]^{-}$} & 363.0635 & -0.5 & $/^{\mathrm{b}}$ & $-c$ \\
\hline & 11 & 6.42 & $\mathrm{C}_{23} \mathrm{H}_{22} \mathrm{O}_{10}$ & 458.1213 & {$\left[\mathrm{M}+\mathrm{HCOO}^{-}\right.$} & 503.1805 & -2.3 & $6 "-O-$ acetyldaidzin & {$[37]$} \\
\hline & 35 & 6.47 & $\mathrm{C}_{13} \mathrm{H}_{10} \mathrm{O}_{6}$ & 262.0466 & {$[\mathrm{M}-\mathrm{H}]^{-}$} & 261.0400 & 2.4 & $/ \mathrm{b}$ & $-c$ \\
\hline & 36 & 6.68 & $\mathrm{C}_{19} \mathrm{H}_{6} \mathrm{~N}_{6} \mathrm{O}_{3}$ & 366.0490 & {$[\mathrm{M}-\mathrm{H}]^{-}$} & 365.0430 & -1.3 & $/ \mathrm{b}$ & $-{ }^{c}$ \\
\hline & 15 & 7.04 & $\mathrm{C}_{23} \mathrm{H}_{22} \mathrm{O}_{11}$ & 474.1162 & {$\left[\mathrm{M}+\mathrm{HCOO}^{-}\right.$} & 519.1784 & 2.3 & $6 "-O-$ acetylgenistin & {$[37]$} \\
\hline & 16 & 7.15 & $\mathrm{C}_{16} \mathrm{H}_{12} \mathrm{O}_{5}$ & 284.0685 & {$[\mathrm{M}-\mathrm{H}]^{-}$} & 283.0969 & 2.1 & Glycitein & [37] \\
\hline & 37 & 7.91 & $\mathrm{C}_{18} \mathrm{H}_{18} \mathrm{O}_{10}$ & 394.0895 & {$\left[\mathrm{M}+\mathrm{HCOO}^{-}\right.$} & 439.0878 & -2.0 & $/ \mathrm{b}$ & $-c$ \\
\hline & 38 & 10.83 & $\mathrm{C}_{31} \mathrm{H}_{50} \mathrm{~N}_{2} \mathrm{O}_{9}$ & 594.3505 & {$[\mathrm{M}-\mathrm{H}]^{-}$} & 593.3490 & -0.6 & b & $-c$ \\
\hline & 39 & 11.29 & $\mathrm{C}_{19} \mathrm{H}_{36} \mathrm{O}_{3}$ & 312.2654 & {$\left[\mathrm{M}-\mathrm{H}-\mathrm{H}_{2} \mathrm{O}\right]^{-}$} & 293.2483 & 1.7 & b & $-c$ \\
\hline & 40 & 11.66 & $\mathrm{C}_{32} \mathrm{H}_{48} \mathrm{~N}_{6} \mathrm{O}_{5}$ & 596.3675 & {$[\mathrm{M}-\mathrm{H}]^{-}$} & 595.3613 & 1.9 & $\rho^{b}$ & $-{ }^{c}$ \\
\hline & 41 & 12.06 & $\mathrm{C}_{17} \mathrm{H}_{36} \mathrm{~N}_{8} \mathrm{O}_{5}$ & 432.2798 & {$[\mathrm{M}-\mathrm{H}]^{-}$} & 431.2728 & 0.1 & $/ \mathrm{b}$ & $-{ }^{c}$ \\
\hline & 42 & 12.54 & $\mathrm{C}_{34} \mathrm{H}_{68} \mathrm{O}_{2}$ & 508.3389 & {$[\mathrm{M}-\mathrm{H}]^{-}$} & 507.3340 & 0.1 & Gheddic acid & [36] \\
\hline & 43 & 12.58 & $\mathrm{C}_{19} \mathrm{H}_{38} \mathrm{O}_{2}$ & 298.2861 & {$[\mathrm{M}-\mathrm{H}]^{-}$} & 297.2814 & 4.0 & Nonadecanoic acid & $-c$ \\
\hline & 44 & 13.57 & $\mathrm{C}_{18} \mathrm{H}_{42} \mathrm{~N}_{10} \mathrm{O}$ & 414.3543 & {$\left[\mathrm{M}+\mathrm{HCOO}^{-}\right.$} & 459.3520 & -1.3 & $/ \mathrm{b}$ & $-{ }^{c}$ \\
\hline & 45 & 15.55 & $\mathrm{C}_{12} \mathrm{H}_{28} \mathrm{~N}_{8} \mathrm{O}_{6}$ & 380.2121 & {$[\mathrm{M}-\mathrm{H}]^{-}$} & 379.2049 & -2.6 & $/^{b}$ & $-{ }^{c}$ \\
\hline
\end{tabular}

Table 2. Mass spectral interpretation of assigned compounds in soybean and Sojae semen praeparatum products.

\begin{tabular}{|c|c|c|}
\hline Compound No. & Assigned Identity (Ion Mode) & MS/MS Fragments Ions \\
\hline 2 & Raffinose (+) & $543.1474[\mathrm{M}+\mathrm{K}]^{+}, 381.0905\left[\mathrm{M}+\mathrm{K}-\mathrm{glu}+\mathrm{H}_{2} \mathrm{O}\right]^{+}$, \\
\hline \multirow{2}{*}{3} & Stachyose $(+)$ & $705.2045[\mathrm{M}+\mathrm{K}]^{+}, 543.1471\left[\mathrm{M}+\mathrm{K}-\mathrm{glu}^{+}\right.$ \\
\hline & $(-)$ & 711.3077[M + HCOO $]^{-}, 665.3014[\mathrm{M}-\mathrm{H}]^{-}, 485.2130[\mathrm{M}-\mathrm{H}-\mathrm{glu}]^{-}, 341.1514\left[\mathrm{M}-\mathrm{H}-2 \mathrm{glu}+2 \mathrm{H}_{2} \mathrm{O}^{-}\right.$ \\
\hline \multirow{2}{*}{5} & $N$-(3-Indolylacetyl)-DL-aspartic acid (+) & $291.0955[\mathrm{M}+\mathrm{H}]^{+}, 161.0645\left[\mathrm{C}_{5} \mathrm{H}_{6} \mathrm{O}_{2} \mathrm{~N}+\mathrm{H}\right]^{+}, 139.0428\left[\mathrm{M}+\mathrm{H}-\mathrm{C}_{8} \mathrm{H}_{6} \mathrm{~N}-2 \mathrm{H}_{2} \mathrm{O}\right]^{+}$ \\
\hline & $(-)$ & $289.1078[\mathrm{M}-\mathrm{H}]^{-}, 271.0968\left[\mathrm{M}-\mathrm{H}-\mathrm{H}_{2} \mathrm{O}\right]^{-}, 245.1127\left[\mathrm{M}-\mathrm{H}-\mathrm{CO}_{2}\right]^{-}, 227.1008\left[\mathrm{M}-\mathrm{H}-\mathrm{CO}_{2}-\mathrm{H}_{2} \mathrm{O}^{-}\right.$, \\
\hline 6 & Daidzin $(+)$ & $439.1128\left[\mathrm{M}+\mathrm{Na}^{+}, 417.1307[\mathrm{M}+\mathrm{H}]^{+}, 277.0551\left[\mathrm{M}+\mathrm{Na}-\text { glu }+\mathrm{H}_{2} \mathrm{O}\right]^{+}, 255.0728\left[\mathrm{M}+\mathrm{H}-\mathrm{glu}+\mathrm{H}_{2} \mathrm{O}^{+}\right.\right.$, \\
\hline 7 & Glycitin $(+)$ & $469.1235[\mathrm{M}+\mathrm{Na}]^{+}, 447.1429[\mathrm{M}+\mathrm{H}]^{+}, 307.0662\left[\mathrm{M}+\mathrm{Na}-\mathrm{glu}+\mathrm{H}_{2} \mathrm{O}\right]^{+}, 285.0839\left[\mathrm{M}+\mathrm{H}-\mathrm{glu}+\mathrm{H}_{2} \mathrm{O}\right]^{+}$ \\
\hline 8 & Genistin (+) & $\begin{array}{l}433.1266\left[\mathrm{M}+\mathrm{H}^{+}, 271.0674\left[\mathrm{M}+\mathrm{H}-\mathrm{glu}+\mathrm{H}_{2} \mathrm{O}\right]^{+}, 243.0712\left[\mathrm{M}+\mathrm{H}-\mathrm{glu}+\mathrm{H}_{2} \mathrm{O}-\mathrm{CO}\right]^{+}, 215.0752[\mathrm{M}+\mathrm{H}-\mathrm{glu}+\right. \\
\left.\mathrm{H}_{2} \mathrm{O}-2 \mathrm{CO}\right]^{+}, 153.0218\left[\mathrm{M}+\mathrm{H}-\mathrm{C}_{13} \mathrm{H}_{12} \mathrm{O}_{7}\right]^{+} \\
477.1629[\mathrm{M}+\mathrm{HCOO}]^{-}, 431.1529\left[\mathrm{M}-\mathrm{H}^{-}, 269.0795\left[\mathrm{M}-\mathrm{H}-\mathrm{glu}+\mathrm{H}_{2} \mathrm{O}\right]^{-}\right.\end{array}$ \\
\hline 9 & $6^{\prime \prime}-O-$ malonyldaidzin $(+)$ & $\begin{array}{l}525.1146[\mathrm{M}+\mathrm{Na}]^{+}, 503.1335[\mathrm{M}+\mathrm{H}]^{+}, 481.1244\left[\mathrm{M}+\mathrm{Na}-\mathrm{CO}_{2}\right]^{+}, 439.1133\left[\mathrm{M}+\mathrm{Na}-\text { malonyl }-\mathrm{H}_{2} \mathrm{O}\right]^{+}, 277.0549[\mathrm{M} \\
+\mathrm{Na}-\text { malonyl } \mathrm{glu}^{+}, 255.0728[\mathrm{M}+\mathrm{H}-\text { malonyl }-\mathrm{glu}]^{+},\end{array}$ \\
\hline
\end{tabular}


Table 2. Cont.

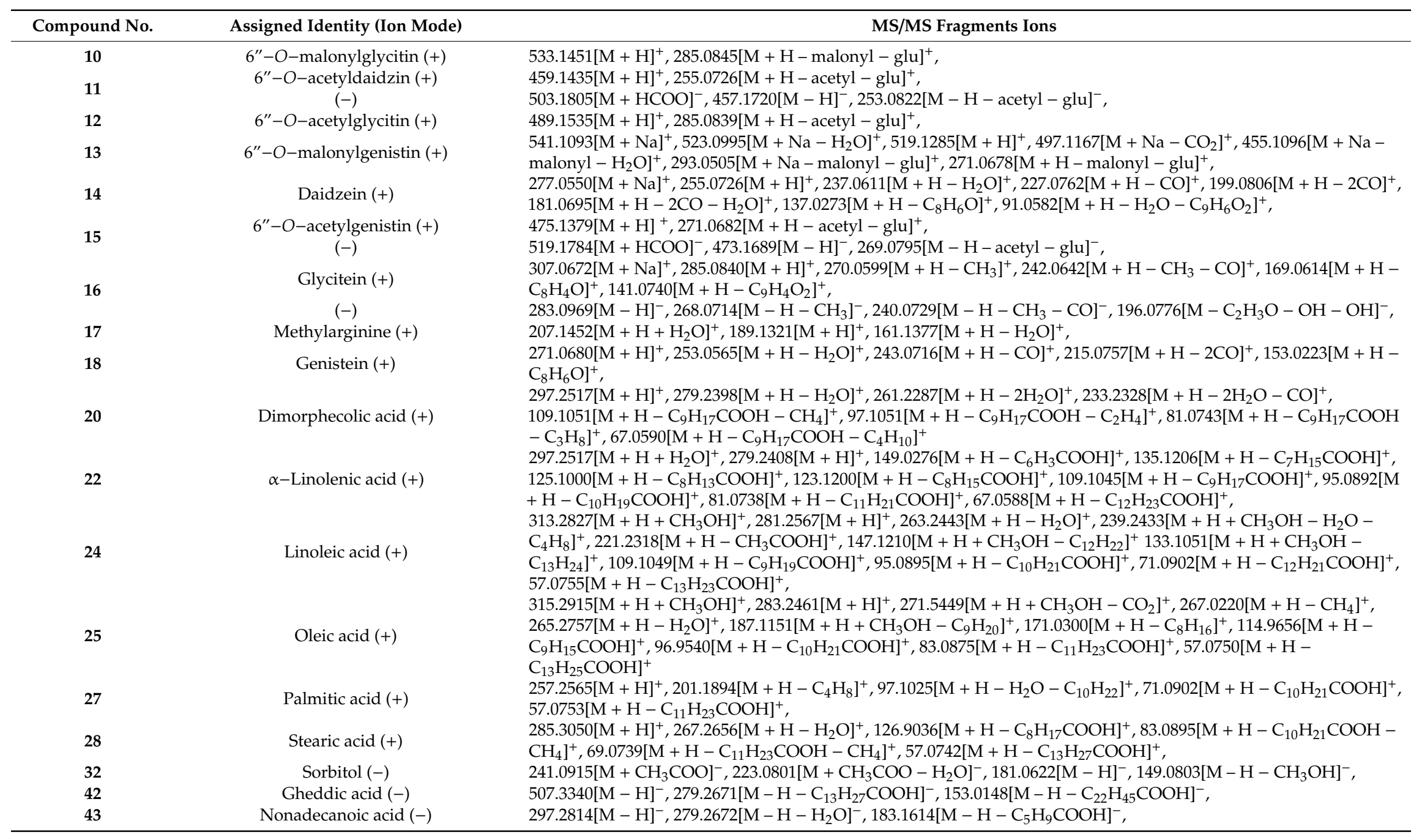



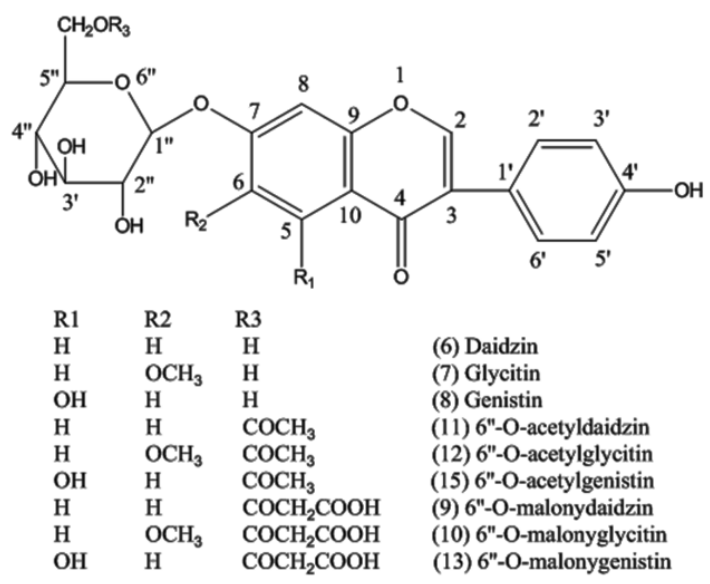

$$
\begin{aligned}
& \text { (6) Daidzin } \\
& \text { (7) Glycitin } \\
& \text { (8) Genistin } \\
& \text { (11) 6"-O-acetyldaidzin } \\
& \text { (12) 6"-O-acetylglycitin } \\
& \text { (15) 6"-O-acetylgenistin } \\
& \text { (9) 6"-O-malonydaidzin } \\
& \text { (10) 6"-O-malonyglycitin } \\
& \text { (13) 6"-O-malonggenistin }
\end{aligned}
$$

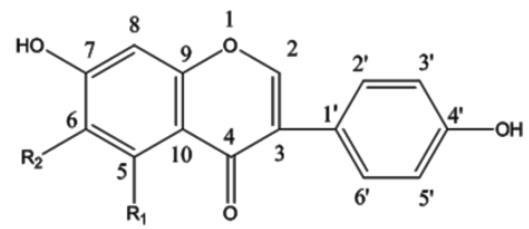

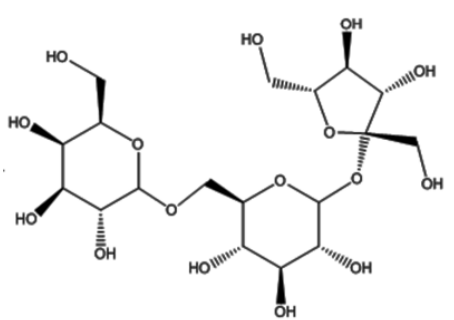

(2) Raffinose

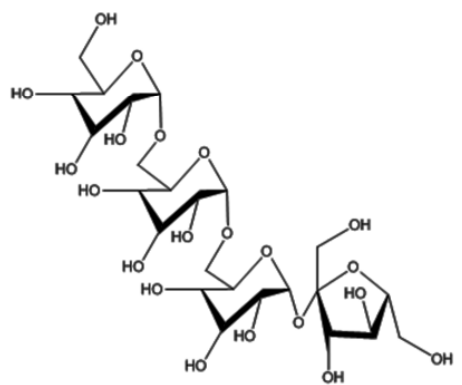

(3) Stachyose

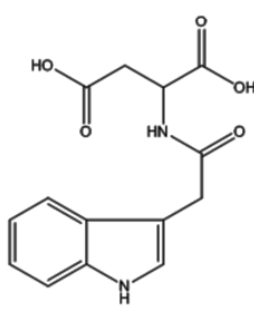

(5) N-(3-Indolylacetyl)-DL-aspartic acid<smiles>CNC(=N)NCCC[C@H](N)C(=O)O</smiles><smiles>CC/C=C/C/C=C/C/C=C/CCCCCCCC(=O)O</smiles>

(22) $\alpha$-Linolenic acid
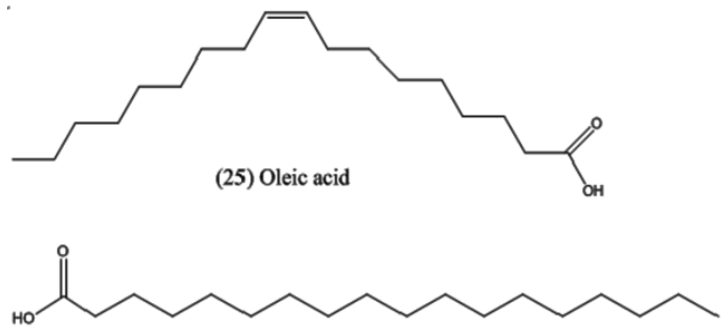

(28) Stearic acid

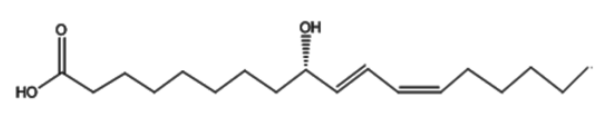

(20) Dimorphecolic acid<smiles>CCCCC/C=C/C/C=C/CCCCCCCC(=O)O</smiles>

(24) Linoleic acid

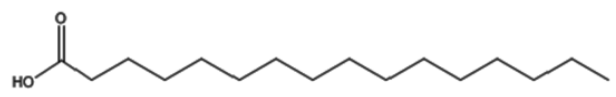

(27) Palmitic acid<smiles>OC[C@@H](O)[C@@H](O)[C@H](O)[C@H](O)CO</smiles>

(32) Sorbitol

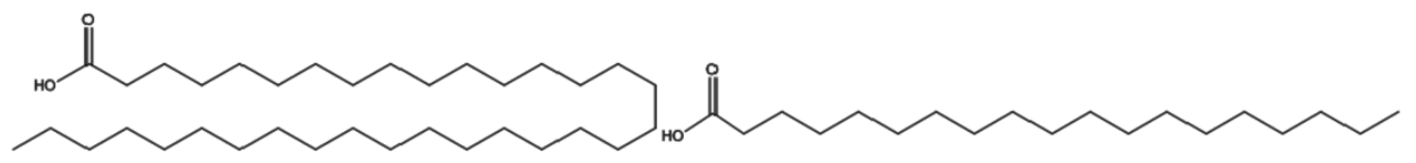

(42) Gheddic acid

(43) Nonadecanoic acid

Figure 4. Chemical structures of assigned compounds.

Compounds 2 and 3 were inferred as raffinose and stachyose, respectively, for the loss of aglyca. Compounds 14, 16 and 18 were putatively identified as isoflavone aglycones for their fragment ions at $m / z$ 137, 153 and 169, respectively, after retro-Diels-Alder reaction. Compounds 6-8 were assigned as isoflavone glycosides for their glycones $\left[\mathrm{M}+\mathrm{H}-\mathrm{glu}+\mathrm{H}_{2} \mathrm{O}\right]^{+}$at $m / z 255,271$ and 285 and $[\mathrm{M}-$ $\left.\mathrm{H}-\mathrm{glu}+\mathrm{H}_{2} \mathrm{O}\right]^{-}$at $m / z$ 253, 269 and 283 after deglycosylation. Further dissociation of the glycones 
yielded a serial of fragments in agreement with the aglycones. The six isoflavone compounds were also confirmed by injecting a mix of standard solutions (Figure 6). Compounds $\mathbf{9 ,} \mathbf{1 0}$ and $\mathbf{1 3}$ were confirmed as isoflavone glycoside malonates: compounds $\mathbf{1 1}, \mathbf{1 2}$ and $\mathbf{1 5}$ were identified as isoflavone acetyl glycosides for their common glycones in comparison with glycosides. Compounds 20, 22, 24, 25, 27 and 28 were assumed to be a series of fatty acids for the homologous fragment ions at $m / z(67,81,95$, 109 and 123); (83, 97 and 111); and (57, 71 and 85), the difference between every pair of the fragment ions was $14\left(-\mathrm{CH}_{2}-\right)$. Twenty of the assigned compounds were previously reported in soybean [33-37]. However, $N$-(3-Indolylacetyl)-DL-aspartic acid, methylarginine, dimorphecolic acid, gheddic acid, and nonadecanoic acid have never been reported in soybean; sorbitol was only detected in germinating soybean seeds [35], and gheddic acid was identified in Mori folium [36]. Six constituents were presumed to be introduced from processing adjuvants or produced during the SSP fermentation process.

\subsection{Relative Quantitative Analysis}

In agreement with previous results [38-40], the relative contents of raffinose and stachyose decreased gradually during the entire fermentation process due to degradation by bacteria. As stachyose and raffinose cause indigestion and flatulence in animals after ingestion, the reduction of oligosaccharides is an indication that fermentation can promote the absorption of soybean nutrients. Isoflavone was inferred to be the principle difference among the products obtained from the SSP fermentation process due to its high proportions of varied components. With increasing fermentation time, the relative contents of isoflavone glycosides decreased while the isoflavone aglycones increased, reaching a maximum in S8. The isoflavone glycoside malonates decreased while the isoflavone acetyl glycosides increased to a maximum in S2 and then dropped. We assumed that the isoflavone glycoside malonates were transformed to acetyl glycosides in the early fermentation period due to their heat instability, and that all the isoflavone glycosides were converted to isoflavone aglycones. The increase in fatty acids showed that lipids could be degraded during fermentation. All the results provide some technological supports for the optimization and quality control of SSP fermentation. Given of the regular component conversion in SSP during fermentation, further identification of the unassigned varied ingredients present in SSP is needed.

\subsection{Accurate Quantitative Analysis}

XIC manager combined with MultiQuant software was used to automatically highlight all findings above a defined thresholds at an exactive mass of $\pm 0.02 \mathrm{Da}$ and to quantitatively compare samples with a series of standard injections.

The validation values are summarized in Table 3. The calibration curves show satisfactory linearity. The correlation coefficient $(r)$ ranged from $0.9840-0.9981$ for all the isoflavones. Limit of detection (LOD) and limit of quantitation (LOQ) values were $0.1-50.0$ and $2.0-250.0 \mathrm{ng} / \mathrm{mL}$, respectively. The intra and inter-day precisions were less than $0.48 \%$ and $2.87 \%$, respectively. The repeatability was within $2.53-4.82 \%$. The recoveries were between $97.61 \pm 3.73 \%$ and $104.84 \pm 2.58 \%$ at different spiking concentration levels. The short-term stability analyzed at various periods was less than $4.10 \%$. The above results demonstrate that the established method is accurate and reproducible for determining the six isoflavones in SSP.

Controlled by mix standard solution, the accurate quantitative results of six isoflavones in SSP were summarized in Figure 5. All six isoflavones were identified in soybean and SSP products. With increasing fermentation time, daidzin, glycitin and genistin decreased while daidzein, glycitein and genistein increased and raised to the top in S8 at 74.50, 13.52 and $47.42 \mathrm{mg} / 100 \mathrm{~g}$ dry weight respectively. Total glycoside and aglycone were also calculated as the sum of each individual isoflavone and presented in Figure 6. As the fermentation time increased, total glycoside contents decreased, while total aglycone contents increased significantly and rose to the top in the S8. The total glycoside content in S8 was less than half a percent of soybean's while the total aglycone content in S8 was 4.8 times higher than that in soybean, indicating that the ingredients converted regularly during the fermentation process. 
Table 3. Validation data of targeted analytes.

\begin{tabular}{|c|c|c|c|c|c|c|c|c|c|c|c|}
\hline \multirow{2}{*}{ Isoflavone } & \multirow{2}{*}{$\begin{array}{l}\mathrm{RT}^{\mathrm{a}} \\
(\mathrm{min})\end{array}$} & \multirow{2}{*}{ Regression Equation ${ }^{b}$} & \multirow{2}{*}{$\begin{array}{c}\text { Linear Range } \\
(\mu \mathrm{g} / \mathrm{mL})\end{array}$} & \multirow{2}{*}{$r$} & \multirow{2}{*}{$\frac{\text { LOD }^{c}}{(\mathrm{ng} / \mathrm{mL})}$} & \multirow{2}{*}{$\frac{\text { LOQ }^{d}}{(\mathrm{ng} / \mathrm{mL})}$} & \multicolumn{2}{|c|}{ Precision (RSD $\%{ }^{e}$ ) } & \multirow{2}{*}{$\begin{array}{l}\text { Repeatability } \\
\text { (RSD, \%) }\end{array}$} & \multirow{2}{*}{$\begin{array}{c}\text { Recovery } \\
\left.\text { (Mean }^{\mathrm{f}} \pm \mathrm{RSD} \%\right)\end{array}$} & \multirow{2}{*}{$\begin{array}{r}\text { Stability } \\
\text { (RSD \% }^{a}\end{array}$} \\
\hline & & & & & & & Intra Day & Inter Day & & & \\
\hline Daidzin & 4.81 & $Y=58544 X+384206$ & $0.010-100.0$ & 0.9979 & 5.0 & 10.0 & 1.14 & 4.24 & 3.98 & $103.83 \pm 3.15$ & 4.10 \\
\hline Glycitin & 5.14 & $Y=49463 X+421236$ & $0.010-100.0$ & 0.9981 & 5.0 & 10.0 & 0.57 & 2.87 & 3.27 & $104.84 \pm 2.58$ & 1.84 \\
\hline Genistin & 5.89 & $Y=60953 X+286019$ & $0.100-600.0$ & 0.9980 & 10.0 & 50.0 & 0.48 & 4.48 & 4.41 & $97.76 \pm 4.70$ & 3.04 \\
\hline Daidzein & 7.02 & $Y=76972 X+705619$ & $0.100-100.0$ & 0.9840 & 0.1 & 3.0 & 1.61 & 3.50 & 2.53 & $97.61 \pm 3.73$ & 3.34 \\
\hline Glycitein & 7.17 & $Y=52697 X+643182$ & $0.010-200.0$ & 0.9973 & 0.1 & 2.0 & 1.42 & 2.61 & 4.19 & $103.71 \pm 2.69$ & 3.78 \\
\hline Genistein & 7.80 & $Y=78899 X+342189$ & $0.50-200.0$ & 0.9973 & 50.0 & 250.0 & 2.35 & 3.99 & 4.82 & $99.67 \pm 3.16$ & 2.39 \\
\hline
\end{tabular}

${ }^{\mathrm{a}} \mathrm{RT}$, retention time; ${ }^{\mathrm{b}} Y$, peak area; $X$, concentration $(\mu \mathrm{g} / \mathrm{mL}) ;{ }^{\mathrm{c}} \mathrm{LOD}$, Limit of detection $(\mathrm{S} / \mathrm{N}=3) ;{ }^{\mathrm{d}} \mathrm{LOQ}$, Limit of quantification $(\mathrm{S} / \mathrm{N}=10) .{ }^{\mathrm{e}}$ Relative standard deviation $(\%)=($ standard deviation $/$ mean $) \times 100$. $(n=3) ;{ }^{\mathrm{f}}$ Mean extraction yield $(\%)=($ detected amount - original amount $) /$ spiked amount $\times 100 .(n=3)$. 
A

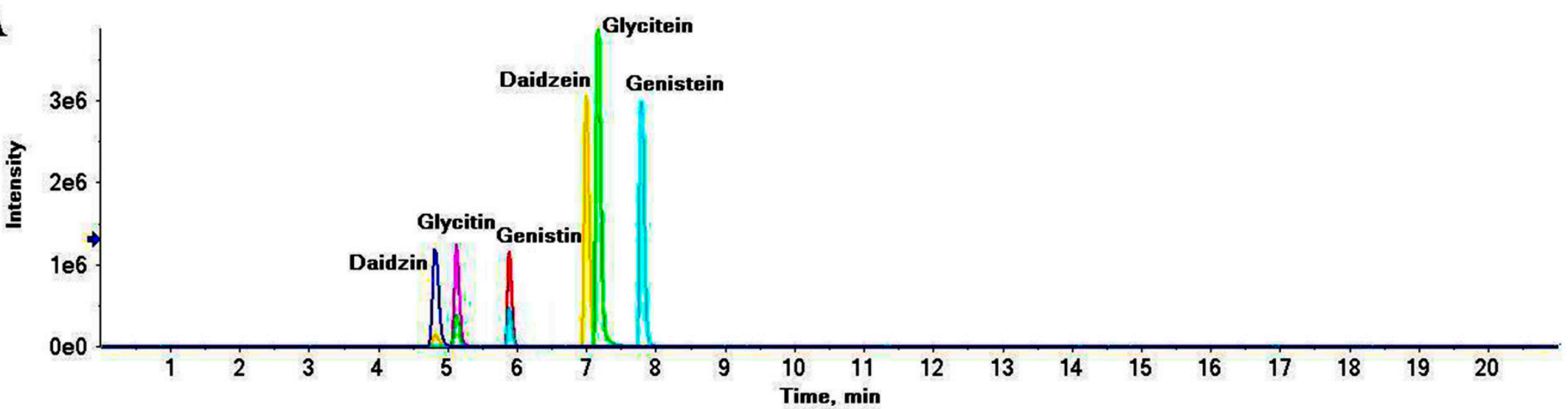

B

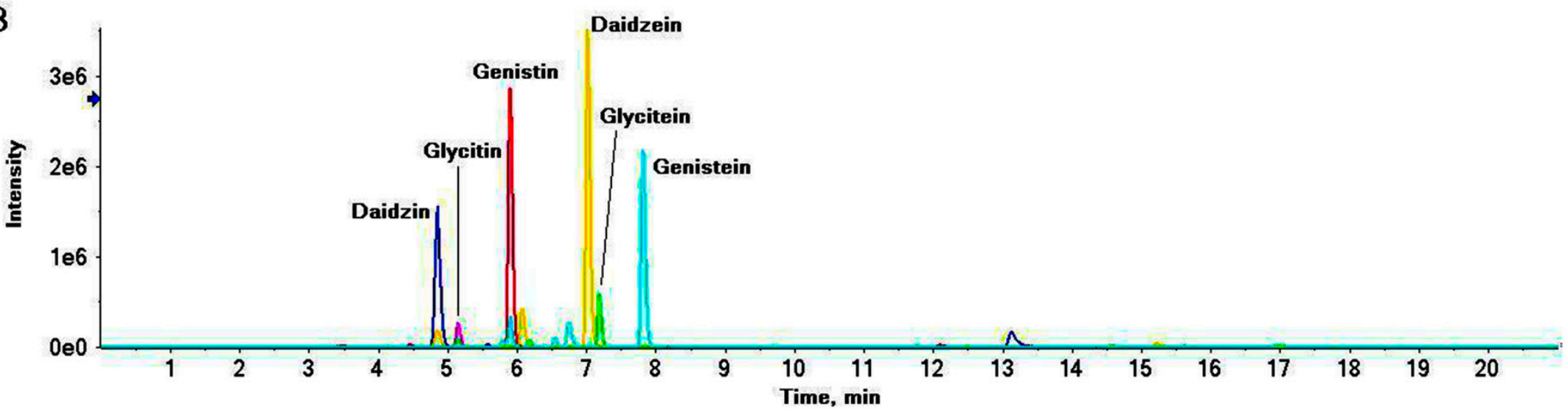

Figure 5. Representative extract ions chromatograms (XIC) of mix standard solution (A) and S8 (B) at $m / z$ 417.118 \pm 0.02 (daidzin), $447.129 \pm 0.02$ (glycitin), $433.113 \pm$ 0.02 (genistin), $255.065 \pm 0.02$ (daidzein), $285.076 \pm 0.02$ (glycitein) and $271.060 \pm 0.02$ (genistein) 


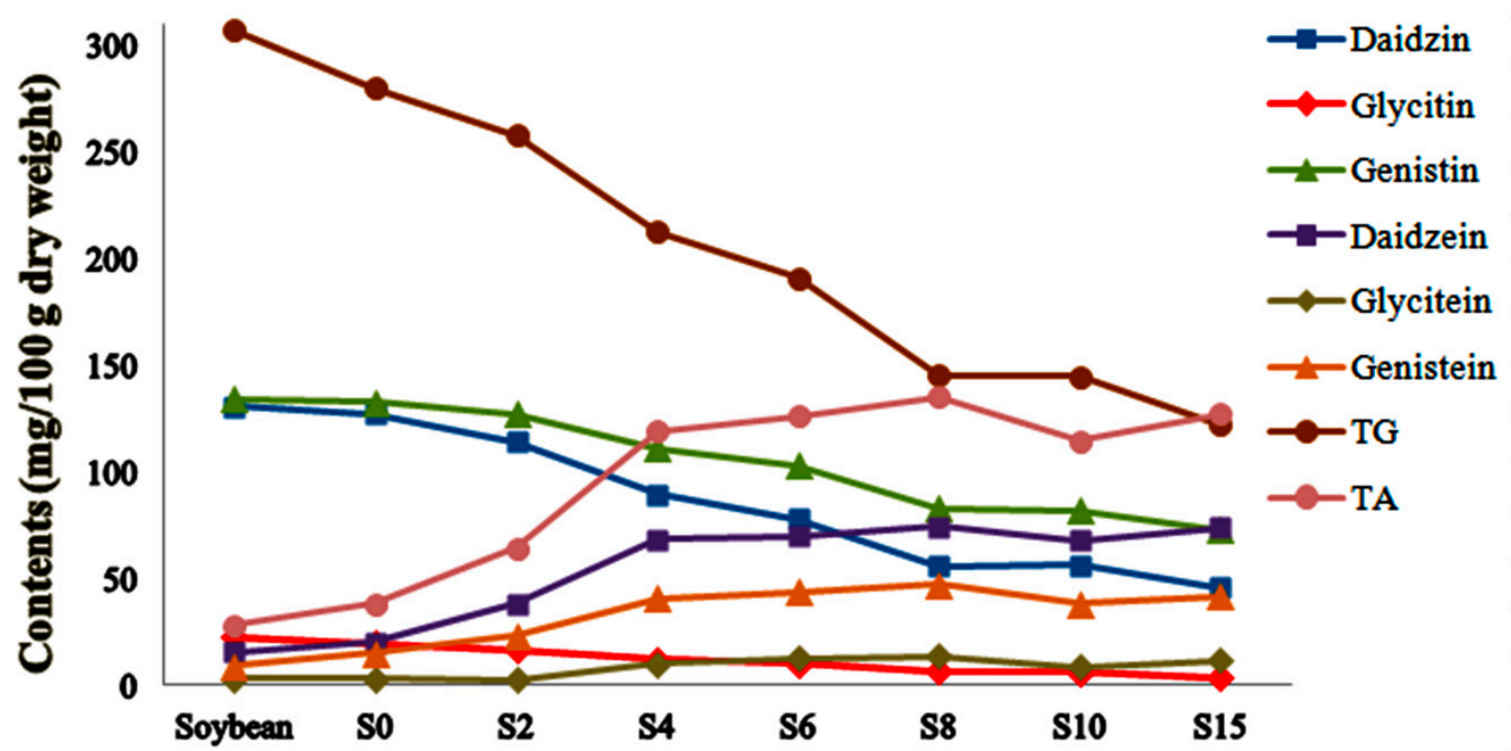

Figure 6. Content profile plots for six isoflavones, total glycoside (TG), and aglycone (TA) in SSP products collected at different fermentation stages.

Previous research has suggested that the increase in aglycone content and $\beta$-glucosidase activity during the fermentation of soybean show a similar trend [41]. As an attractive candidate to convert isoflavone glycosides to their aglycones, $\beta$-glucosidase reached its maximum activity on the eighth days of fermentation.

As aglycone possesses higher bioactivity and bioavailability compared to the $\beta$-glycosides isoflavone, the quantitative results of the representative constituents illustrated that eight days is the optimal time for the fermentation of SSP, which agrees with the morphologic changes (Figure 1). We reasoned that the bioactivities may be related to the variations in isoflavone content and $\beta$-glucosidase activity during SSP fermentation. Moreover, we created a perfect setup for SSP fermentation quality assessment and quality control. To determine the impact of bacterial or fungi on SSP fermentation, -further study is required.

\section{Materials and Methods}

\subsection{Chemicals and Reagents}

The reference standards of daidzin, glycitin, genistin, daidzein, glycitein and genistein were all purchased from Sigma-Aldrich (St. Louis, MO, USA)

Liquid chromatography (LC)/MS-grade acetonitrile, formic acid, methanol, and water were purchased from Merck Co. (Darmstadt, Germany).

Soybean, Artemisiae annuae herba and Mori folium used in the fermentation were purchased from YiFeng TCM shop (Nanjing, China) and authenticated by Associate Professor Jianwei Chen (Department of Pharmacy, Nanjing University of Chinese Medicine, Nanjing, Jiangsu, China).

\subsection{SSP Fermentation}

SSP was fermented in the laboratory and the preparation was performed as described in detail by the 2015 edition of China Pharmacopoeia as illustrated in Figure 7. The steps and parameters were as follows: 


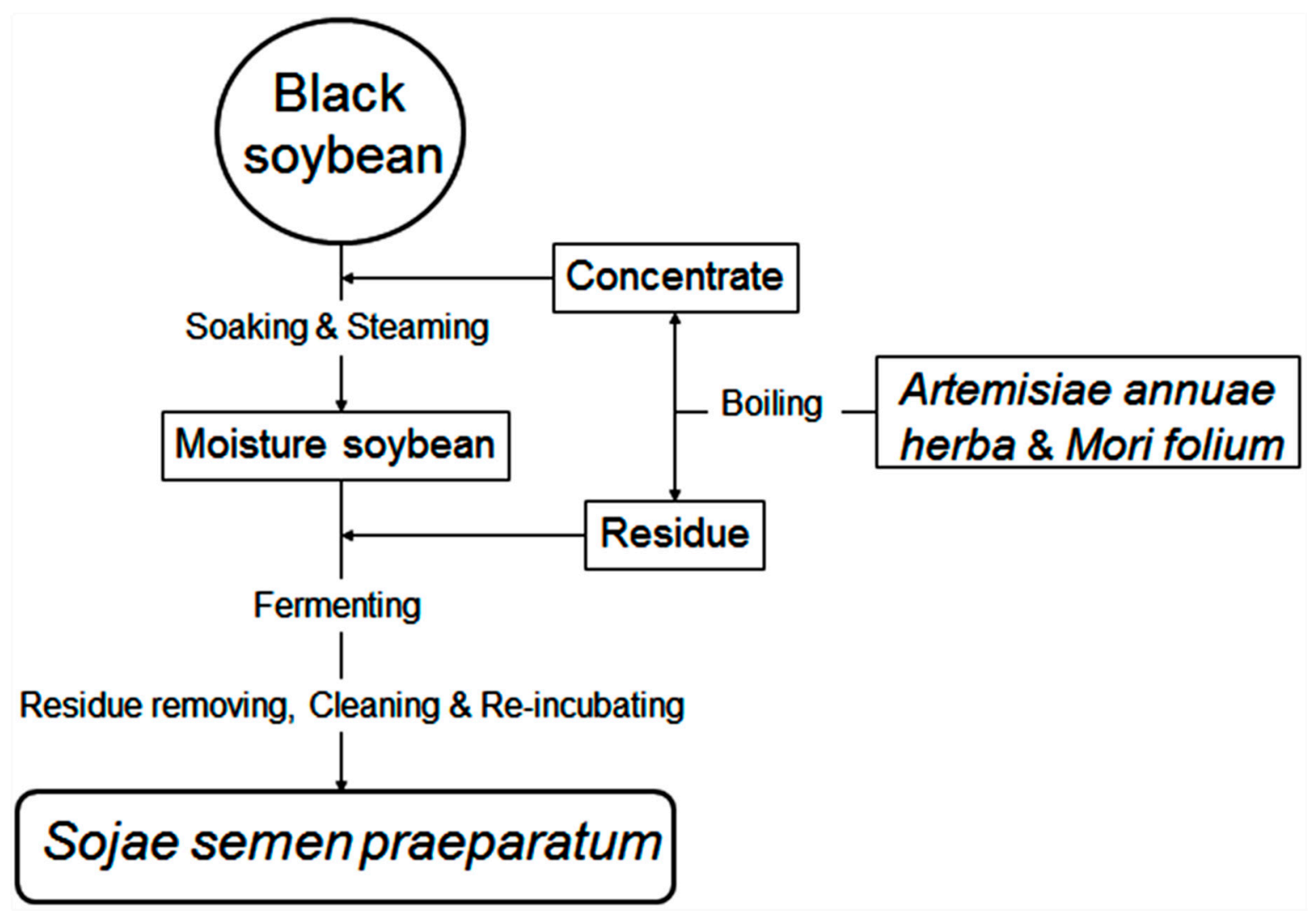

Figure 7. Flow diagram for fermentation of soybean to Sojae semen praeparatum (SSP).

Artemisiae annuae herba (100 g dry weight) and Mori folium (90 g dry weight) were washed and boiled with water $(3600 \mathrm{~mL})$ for $1 \mathrm{~h}$ in triplicate, and the decoction was concentrated to a relative density of $1.10-1.12 \mathrm{~g} / \mathrm{cm}^{3}$ concentrate. Black soybean (1000 g dry weight) was soaked in the concentrate overnight and steamed for $1.5 \mathrm{~h}$ while covered with wet cheesecloth. Aliquots (100 g wet weight) of the moisture soybean were placed on enamel trays covered with wet cheesecloth, which were covered with the residue of boiled Artemisiae annuae herba and Mori folium and incubated at $37^{\circ} \mathrm{C}$ with $60-80 \%$ humidity. After fermenting for $0,2,4,6,8,10,15$ days and removal of residues, wash cleaning, and re-incubating until a sweet smell drifting out, the SSP samples (S0, S2, S4, S6, S8, S10, S12, and S15) were then dried and pulverized into powder using an electric mill and sieved through 80 mesh sieves.

\subsection{Sample Extraction}

One gram of the powdered samples was accurately weighed and extracted with $25 \mathrm{~mL}$ of $75 \%$ methanol at $80{ }^{\circ} \mathrm{C}$ for $30 \mathrm{~min}$ using a Soxhlet extractor. This was followed by centrifugation for $15 \mathrm{~min}$ at $12000 \mathrm{rpm}$. The extraction supernatants were then diluted 10 times and filtered through a $0.45 \mu \mathrm{m}$ filter unit.

\subsection{Standard Solutions Preparation}

Mix standard solutions were prepared by accurately weighing the standard substances and mixing them in $75 \%$ methanol. This standard mixture was filtered through a $0.45 \mu \mathrm{m}$ filter unit.

\subsection{LC-MS Spectrometric Conditions}

An ultra-fast liquid chromatography system (Shimadzu Corporation UFLC XR; Kyoto, Japan) was connected to a triple time-of-flight mass spectrometer (TripleTOF 5600 system, AB SCIEX, Los Angeles, CA, USA) with an electrospray ionization source. 
All samples were separated by an ACQUITY UPLC BEH C 18 column $(2.1 \mathrm{~mm} \times 100 \mathrm{~mm}, 1.7$ $\mu \mathrm{m}$, Waters Corp., Milford, MA, USA). A binary solvent gradient consisting of solvent A (water with $0.2 \%$ formic acid) and solvent B (acetonitrile with $0.2 \%$ formic acid) was used. The flow rate was 300 $\mu \mathrm{L} / \mathrm{min}$. The total run time was $21 \mathrm{~min}$ with a gradient as follows: $0-3 \mathrm{~min}, 10-16 \% \mathrm{~B} ; 3-7 \mathrm{~min}, 16-50 \%$ B; 7-12 $\min , 50-80 \%$ B; 12-15 $\min , 80-90 \%$ B; 15-17 $\min , 90-10 \%$ B; and 17-21 min, 10\% B for column equilibration before the next run. An injection volume of $1 \mu \mathrm{L}$ was used. The column temperature was $40^{\circ} \mathrm{C}$.

The samples were analyzed by acquiring full scan MS data in both positive and negative ion modes. The automatic data-dependent information product ion spectra (IDA-MS/MS) without any predefinition of the ions was checked. A calibrated delivery system was used to ensure the accuracy error of masses less than $1 \mathrm{ppm}$. The settings were nitrogen gas for nebulization at 55 psi, heater gas pressure at 55 psi, curtain gas at 35 psi, temperature of $500{ }^{\circ} \mathrm{C}$, and ion spray voltage at $5500 \mathrm{~V}$ in positive ion mode, and $-4500 \mathrm{~V}$ in negative ion mode. The acquisition of a survey tripleTOF MS spectrum was operated under high-resolution settings. The optimized declustering potential (DP) and collision energy (CE) were set at $80 \mathrm{eV}$ and $15 \mathrm{eV}$ in positive ion mode, and to $-80 \mathrm{eV}$ and $-15 \mathrm{eV}$ in negative ion mode, respectively. A sweeping collision energy setting at 35/-35 eV $\pm 15 \mathrm{eV}$ was applied for collision-induced dissociation (CID).

\subsection{Method Validation}

The method was fully validated in accordance with guidelines on linearity, precision, recovery, detection limit, quantification limit, and stability. Calibration curves were generated by plotting peak area against the concentration of standard solutions. The intra-day precision was examined for six replicates of injections with the mixed standard solutions in one day, and the inter-precision was determined by injection in duplicates over three consecutive days. All the results are expressed using the relative standard deviation (RSD). The LOD and LOQ were calculated based on the peak-to-noise ratios of 3:1 and 10:1, respectively. The repeat, recovery, and stability were tested on the analytes in S8, and the repeatability was analyzed on six sample solutions from the same sample in parallel. The recovery was used to evaluate the accuracy at different spiking concentration levels (80\%, 100\%, and $120 \%$ as compared to the nominal concentration) of standard solutions. and the sample stability was tested by periodic analysis at room temperature for various periods $(0,2,4,8,12,16,20$, and $24 \mathrm{~h})$.

\subsection{Data Processing}

TOF-MS data were collected using Analyst ${ }^{\circledR}$ version 1.6 software (AB SCIEX, Los Angeles, CA, USA) and processed using PeakView ${ }^{\circledR}$ version 1.2 software (AB SCIEX, Los Angeles, CA, USA) with the XIC Manager (AB SCIEX, Los Angeles, CA, USA) add-in and MultiQuant ${ }^{\mathrm{TM}}$ version 2.1 software (AB SCIEX, Los Angeles, CA, USA).

The PeakView ${ }^{\circledR}$ software contained a simple fragment ion predictor to help link the MS/MS spectrum to structures (saved as .mol files) and to provide insights into fragmentation mechanisms.

XIC Manager was used for targeted and non-targeted data processing, which consisted of a table for defining a list of masses or formulae to generate extracted ion chromatograms (XIC), and to review the identification of detected compounds. Our high confidence in results is based on retention times, accurate mass, isotopic pattern and MS/MS library searching.

The PCA was performed using MarkerView ${ }^{\circledR}$ software, where three repeated spectra for each sample were imported and analyzed with Pareto scaling. The $T$-value and corresponding $P$-value were calculated between each group and all the other eight groups. The program was linked back to the raw data so that differences could be directly visualized in spectra or chromatograms. The converted components were putatively identified by PeakView ${ }^{\circledR}$ software. 


\section{Conclusions}

This was the first systematic comparison of active ingredients in the raw materials and processed products obtained during Sojae semen praeparatum (SSP) fermentation. Simultaneous characterization and quantification were performed using ultra-fast liquid chromatography (UFLC)-TripleTOF MS combined with XIC manager. The quantitative results verified that the components converted during the SSP fermentation, and we identified 45 components in positive ion mode and negative ion mode, in which 25 were putatively identified and a high proportion was isoflavone. N-(3-Indolylacetyl)-DL-aspartic acid, methylarginine, dimorphecolic acid, sorbitol, gheddic acid, and nonadecanoic acid were presumed to be introduced from processing adjuvants or produced during the fermentation process. The relative contents of raffinose and stachyose decreased gradually, while the fatty acids and isoflavone aglycones increased, which indicated that fermentation promotes the absorption of soybean nutrition and lipids degradation. The accurate quantitation of isoflavone, the representative constituents in soybean, revealed that fermentation for eight days produced a marked increase in the content of aglycone, the bioactive isoflavone, and a significant reduction in the content of $\beta$-glycosides isoflavone compared with unfermented soybean. This illustrated that eight days is the optimal time for the fermentation of SSP from the aspects of content, in agreement with the morphologic changes. We reasoned that the bioactivities of SSP might be related to isoflavone. Our study has provided some technological support for the optimization and quality control of SSP fermentation.

Supplementary Materials: The following are available online, Figure S1: Selected ion intensity trend plots of assigned identities., Figure S2: Mass spectrum of assigned compounds in soybean and Semen sojae praeparatum products.

Author Contributions: Conceptualization, C.C., X.C. and H.W.; data curation, C.C. and C.S.; formal analysis, C.C. and C.S.; funding acquisition, H.W.; methodology, C.C.; resources, S.Y. and H.W.; software, C.C. and X.W.; supervision, X.C. and S.Y.; validation, C.C.; writing—original draft, C.C.; writing-review \& editing, H.W.

Funding: This work was supported by National Natural Science Foundation of China (51403104), Jiangsu Key Laboratory for Functional Substance of Chinese Medicine, the Natural Science Foundation of the Jiangsu Higher Education Institutions of China (08KJB360004), School Foundation of Nanjing University of Chinese Medicine, the People's Republic of China under Grant No. 12XZR24 and A Project Funded by the Priority Academic Program Development of Jiangsu Higher Education Institutions, Top-notch Academic Programs Project of Jiangsu Higher Education Institutions (TAPP-PPZY2015A070).

Conflicts of Interest: The authors declare no conflicts of interest.

\section{References}

1. Choi, M.-Y.; Chai, C.; Park, J.H.; Lim, J.; Lee, J.; Kwon, S.W. Effects of storage period and heat treatment on phenolic compound composition in dried citrus peels (chenpi) and discrimination of chenpi with different storage periods through targeted metabolomic study using hplc-dad analysis. J. Pharm. Biomed. Anal. 2011, 54, 638-645. [CrossRef]

2. Mortera, P.; Zuljan, F.A.; Magni, C.; Bortolato, S.A.; Alarcon, S.H. Multivariate analysis of organic acids in fermented food from reversed-phase high-performance liquid chromatography data. Talanta 2018, 178, 15-23. [CrossRef] [PubMed]

3. Chai, C.; Ju, H.K.; Kim, S.C.; Park, J.H.; Lim, J.; Kwon, S.W.; Lee, J. Determination of bioactive compounds in fermented soybean products using $\mathrm{gc} / \mathrm{ms}$ and further investigation of correlation of their bioactivities. J. Chromatogr. B 2012, 880, 42-49. [CrossRef] [PubMed]

4. Zhu, H.; Bi, K.; Han, F.; Guan, J.; Zhang, X.; Mao, X.; Zhao, L.; Li, Q.; Hou, X.; Yin, R. Identification of the absorbed components and metabolites of zhi-zi-da-huang decoction in rat plasma by ultra-high performance liquid chromatography coupled with quadrupole-time-of-flight mass spectrometry. J. Pharm. Biomed. Anal. 2015, 111, 277-287. [CrossRef]

5. Zhang, P.; Zhang, P.; Xie, M.; An, F.; Qiu, B.; Wu, R. Metaproteomics of microbiota in naturally fermented soybean paste, da-jiang. J. Food Sci. 2018, 83, 1342-1349. [CrossRef] [PubMed]

6. Kim, M.S.; Kim, B.; Park, H.; Ji, Y.; Holzapfel, W.; Kim, D.Y.; Hyun, C.K. Long-term fermented soybean paste improves metabolic parameters associated with non-alcoholic fatty liver disease and insulin resistance in high-fat diet-induced obese mice. Biochem. Biophys. Res. Commun. 2018, 495, 1744-1751. [CrossRef] [PubMed] 
7. Ali, M.W.; Shahzad, R.; Bilal, S.; Adhikari, B.; Kim, I.D.; Lee, J.D.; Lee, I.J.; Kim, B.O.; Shin, D.H. Comparison of antioxidants potential, metabolites, and nutritional profiles of korean fermented soybean (cheonggukjang) with bacillus subtilis kctc 13241. J. Food Sci. Technol. 2018, 55, 2871-2880. [CrossRef]

8. Qu, L.; Fan, G.; Peng, J.; Mi, H. Isolation of six isoflavones from semen sojae praeparatum by preparative HPLC. Fitoterapia 2007, 78, 200-204. [CrossRef] [PubMed]

9. Chai, C.; Bai, Y.T.; Wen, H.M.; Song, L.H.; Tu, J.Y.; Shan, C.X. Determination of 3 kinds of isoflavone aglycones in semen sojae praeparatum by uplc. Chin. J. Ethnomed. Ethnopharm. 2012, 12, 2.

10. Guo, H.; Zhang, Z.; Yao, Y.; Liu, J.; Chang, R.; Liu, Z.; Hao, H.; Huang, T.; Wen, J.; Zhou, T. A new strategy for statistical analysis-based fingerprint establishment: Application to quality assessment of semen sojae praeparatum. Food Chem. 2018, 258, 189-198. [CrossRef] [PubMed]

11. Qiu, F.; Shi, L.; Wang, S.; Wu, S.; Wang, M. Simultaneous high-performance liquid chromatography with diode array detection and time-of-flight mass spectrometric confirmation of the ten bioactive compounds in semen sojae preparatum. J. Sep. Sci. 2018, 41, 3360-3371. [CrossRef]

12. Zhang, J.-H.; Tatsumi, E.; Ding, C.-H.; Li, L.-T. Angiotensin i-converting enzyme inhibitory peptides in douchi, a chinese traditional fermented soybean product. Food Chem. 2006, 98, 551-557. [CrossRef]

13. Tsai, Y.-H.; Kung, H.-F.; Chang, S.-C.; Lee, T.-M.; Wei, C.-I. Histamine formation by histamine-forming bacteria in douchi, a chinese traditional fermented soybean product. Food Chem. 2007, 103, 1305-1311. [CrossRef]

14. Chai, C.; Cui, X.; Shan, C.; Yu, S.; Wen, H. Contents variation analysis of free amino acids, nucleosides and nucleobases in semen sojae praeparatum fermentation using uflc-qtrap ms. Biomed. Chromatogr. 2017, 31, 1-11. [CrossRef]

15. Chai, C.; Yu, S.; Cui, X.B.; Zhang, A.H.; Zhu, D.; Shan, C.X.; Wen, H.M. Analysis of volatile components in semen sojae praepatum with automatic static headspace and gas chromatography-mass spectrometry. Food Res. Dev. 2013, 34, 4 .

16. Suo, H.; Feng, X.; Zhu, K.; Wang, C.; Zhao, X.; Kan, J. Shuidouchi (fermented soybean) fermented in different vessels attenuates hcl/ethanol-induced gastric mucosal injury. Molecules 2015, 20, 19748-19763. [CrossRef]

17. Qu, K.; Zhao, L.; Luo, X.; Zhang, C.; Hou, P.; Bi, K.; Chen, X. An LC-MS method for simultaneous determination of five iridoids from zhi-zi-chi decoction in rat brain microdialysates and tissue homogenates: Towards an in depth study for its antidepressive activity. J. Chromatogr. B Anal. Technol. Biomed. Life Sci. 2014, 965, 206-215. [CrossRef]

18. Chen, J.; Cheng, Y.-Q.; Yamaki, K.; Li, L.-T. Anti- $\alpha$-glucosidase activity of chinese traditionally fermented soybean (douchi). Food Chem. 2007, 103, 1091-1096. [CrossRef]

19. McClean, S.; Beggs, L.B.; Welch, R.W. Antimicrobial activity of antihypertensive food-derived peptides and selected alanine analogues. Food Chem. 2014, 146, 443-447. [CrossRef]

20. Chen, L.; Liu, Q.; Sun, Y.; Wang, P.; Zhang, L.; Wang, W.J.C.P. Screening and enzymatic activity analysis of dominant fermentive bacteria of sojae semen praeparatum from different production places. China Pharma. 2017, 28, 4359-4361.

21. Li, G.; Long, K.; Su, M.S.; Liang, Y.H.; Yang, A.J.; Xie, X.M. Preliminary study on dynamic change of microbial flora in fermentation process to 'yellow cladding' of sojoe semen praeparatum. Chin. J. Exp. Tradit. Med. Formulae 2014, 20, 139-142.

22. Blay, M.; Espinel, A.E.; Delgado, M.A.; Baiges, I.; Bladé, C.; Arola, L.; Salvadó, J. Isoflavone effect on gene expression profile and biomarkers of inflammation. J. Pharm. Biomed. Anal. 2010, 51, 382-390. [CrossRef]

23. Kao, T.H.; Chen, B.H. Effects of different carriers on the production of isoflavone powder from soybean cake. Molecules 2007, 12, 917-931. [CrossRef]

24. Saha, J.; Biswas, A.; Chhetri, A.; Sarkar, P.K. Response surface optimisation of antioxidant extraction from kinema, a bacillus-fermented soybean food. Food Chem. 2011, 129, 507-513. [CrossRef]

25. Wang, L.; Yin, L.; Li, D.; Zou, L.; Saito, M.; Tatsumi, E.; Li, L. Influences of processing and nacl supplementation on isoflavone contents and composition during douchi manufacturing. Food Chem. 2007, 101, 1247-1253. [CrossRef]

26. Hurtado-Fernández, E.; Gómez-Romero, M.; Carrasco-Pancorbo, A.; Fernández-Gutiérrez, A. Application and potential of capillary electroseparation methods to determine antioxidant phenolic compounds from plant food material. J. Pharm. Biomed. Anal. 2010, 53, 1130-1160. [CrossRef] 
27. Gaya, P.; Medina, M.; Sanchez-Jimenez, A.; Landete, J.M. Phytoestrogen metabolism by adult human gut microbiota. Molecules 2016, 21, 1034. [CrossRef]

28. Chung, I.-M.; Seo, S.-H.; Ahn, J.-K.; Kim, S.-H. Effect of processing, fermentation, and aging treatment to content and profile of phenolic compounds in soybean seed, soy curd and soy paste. Food Chem. 2011, 127, 960-967. [CrossRef]

29. Yang, S.; Wang, L.; Yan, Q.; Jiang, Z.; Li, L. Hydrolysis of soybean isoflavone glycosides by a thermostable $\beta$-glucosidase from paecilomyces thermophila. Food Chem. 2009, 115, 1247-1252. [CrossRef]

30. Kaya, M.; Ito, J.; Kotaka, A.; Matsumura, K.; Bando, H.; Sahara, H.; Ogino, C.; Shibasaki, S.; Kuroda, K.; Ueda, M.; et al. Isoflavone aglycones production from isoflavone glycosides by display of beta-glucosidase from aspergillus oryzae on yeast cell surface. Appl. Microbiol. Biotechnol. 2008, 79, 51-60. [CrossRef]

31. Zeng, X.; Su, W.; Zheng, Y.; Liu, H.; Li, P.; Zhang, W.; Liang, Y.; Bai, Y.; Peng, W.; Yao, H. UFLC-Q-TOF-MS/ MS-based screening and identification of flavonoids and derived metabolites in human urine after oral administration of exocarpium citri grandis extract. Molecules 2018, 23, 895. [CrossRef] [PubMed]

32. The Pharmacopoeia Commission of the Ministry of Health of the People's Republic of China. Pharmacopoeia of People's Republic of China; Part I; Medical Science and Technology Press: Beijing, China, 2015; p. 328.

33. Huang, Y.; Zhang, H.; Ben, P.; Duan, Y.; Lu, M.; Li, Z.; Cui, Z. Characterization of a novel gh36 alpha-galactosidase from bacillus megaterium and its application in degradation of raffinose family oligosaccharides. Int. J. Biol. Macromol. 2018, 108, 98-104. [CrossRef]

34. Yu, F.X.; Chen, X.; Chen, Z.W.; Wei, X.J. Fatty acid analysis of edible oils. Adv. Mater. Res. 2014, 962-965, 1222-1225. [CrossRef]

35. Kuo, T.M.; Doehlert, D.C.; Crawford, C.G. Sugar metabolism in germinating soybean seeds. Evidence for the sorbitol pathway in soyabean axes. Plant Physiol. 1990, 93, 1514-1520. [CrossRef] [PubMed]

36. Wang, X.J.; Wang, Y.S.; Qiu, J. Study on chemical composition of folium mori. Food Drug 2007, 30, 1-3.

37. Li, S.; Li, S.; Liu, C.; Liu, C.; Zhang, Y. Extraction and isolation of potential anti-stroke compounds from flowers of pueraria lobata guided by in vitro pc12 cell model. J. chromatogr. B Anal. Technol. Biomed. Life Sci. 2017, 1048, 111-120. [CrossRef] [PubMed]

38. Yang, Y.J.; Yao, Y.S.; Qin, Y.C.; Qiu, J.; Li, J.G.; Li, J.; Gu, X. Investigation and analysis of main afn in soybean meal and fermented soybean meal. Sci. Agric. Sin. 2016, 49, 573-580.

39. Liu, Y.C.; Cao, Y.; Zhang, J.X.; Zhang, N.; Lin-Jie, B.I.; Liu, T.; Chen, J.J. The condition optimization of bacterial combinations for fermentation of bean and determination of the content of stachyose and raffinose. Hubei Agric. Sci. 2015, 54, 4007-4011.

40. Fu, T.; Qi, W.; Li, A.; Liu, J.; Liang, X.; Yun, T.; Wang, Y. Effects of different bacteria on fermentative degradation of stachyose and raffinose in soybean meal and detection technology. J. Chin. Cereals Oils Assoc. 2014, 29, 111-118.

41. Lee, I.H.; Chou, C.C. Distribution profiles of isoflavone isomers in black bean kojis prepared with various filamentous fungi. J. Agric. Food Chem. 2006, 54, 1309-1314. [CrossRef]

Sample Availability: Samples of the compounds are available from the authors.

(C) 2019 by the authors. Licensee MDPI, Basel, Switzerland. This article is an open access article distributed under the terms and conditions of the Creative Commons Attribution (CC BY) license (http://creativecommons.org/licenses/by/4.0/). 